\section{Frequency division using a soliton-injected semiconductor gain-switched frequency comb}

\author{
Wenle Weng ${ }^{1 *}$, Aleksandra Kaszubowska-Anandarajah ${ }^{2 *}$, Junqiu Liu ${ }^{1}$, \\ Prince M. Anandarajah ${ }^{3}$, Tobias J. Kippenberg ${ }^{1 *}$
}

\begin{abstract}
With optical spectral marks equally spaced by a frequency in the microwave or the radio frequency domain, optical frequency combs have been used not only to synthesize optical frequencies from microwave references but also to generate ultralow-noise microwaves via optical frequency division. Here, we combine two compact frequency combs, namely, a soliton microcomb and a semiconductor gain-switched comb, to demonstrate low-noise microwave generation based on a novel frequency division technique. Using a semiconductor laser that is driven by a sinusoidal current and injection-locked to microresonator solitons, our scheme transfers the spectral purity of a dissipative soliton oscillator into the subharmonic frequencies of the microcomb repetition rate. In addition, the gain-switched comb provides dense optical spectral emissions that divide the line spacing of the soliton microcomb. With the potential to be fully integrated, the merger of the two chipscale devices may profoundly facilitate the wide application of frequency comb technology.
\end{abstract}

\section{INTRODUCTION}

Self-referenced optical frequency combs (OFCs) based on femtosecond pulse lasers have provided phase-coherent links between the optical domain and the radio frequency (RF) or microwave domain $(1,2)$. On the one hand, these mode-locked lasers can synthesize frequencies that span from extreme-ultraviolet (3) to mid-infrared ranges (4) with $\mathrm{RF} /$ microwave references such as atomic clocks (5-7) or stable masers $(8,9)$, thus revolutionizing the fields of optical metrology and spectroscopy (10). On the other hand, owing to the development of the frequency division technique that transfers the spectral purity of an optical reference to the microwave domain with the benefit of a divided phase noise spectral density, the photodetection of pulse trains has been used to synthesize microwaves with the lowest phase noise levels achieved $(11,12)$. Yet, despite their exquisite performance, to date, the use of mode-locked laser-based OFCs is mostly limited to the laboratory environment due to the bulky sizes, the high power consumption, and their delicate structures.

To overcome the difficulties of out-of-laboratory usage of traditional OFCs and to meet the urgent demand of low-cost, portable, and power-efficient comb sources that are suitable for field-deployable time keeping, navigation, and high-bitrate telecommunication, alternative OFC techniques have rapidly advanced in the past decade (13-15). Among them, two approaches have attracted significant research interest. The first is the microresonator frequency combs based on dissipative Kerr solitons (DKS) (16). Also referred to as soliton microcombs, these OFCs are generated with self-organization of localized light structures in Kerr microresonators driven by continuous-wave $(\mathrm{CW})$ lasers. With their high repetition rates and intrinsic high coherence, soliton microcombs have been applied in ranging $(17,18)$, low-noise RF generation (19), and integrated clocks

\footnotetext{
'Laboratory of Photonics and Quantum Measurements (LPQM), Swiss Federal Institute of Technology Lausanne (EPFL), CH-1015 Lausanne, Switzerland. ${ }^{2}$ CONNECT Research Centre, Dunlop Oriel House, Trinity College Dublin, Dublin 2, Ireland. ${ }^{3}$ Photonics Systems and Sensing Lab., School of Electronic Engineering, Dublin City University, Glasnevin D 9, Ireland.

*Corresponding author. Email: wenle.weng@epfl.ch (W.W.); anandara@tcd.ie (A.K.-A.); tobias.kippenberg@epfl.ch (T.J.K.)
}

(20). The second approach uses semiconductor gain-switched lasers (GSLs) to create laser pulse trains by rapidly switching the gain above and below the lasing threshold $(21,22)$. With the gain switching frequency being externally controlled by a microwave signal, these pulsed lasers are highly flexible and tunable in both the repetition rate and the emission wavelength (23-26). Successful applications of GSLs have been demonstrated in quantum key distribution (27), random number generation (28), and coherent optical communication $(29,30)$. However, compared to mode-locked laser OFCs, GSL OFCs have very narrow optical spans that are usually of a few nanometers. Moreover, GSL OFCs rely on auxiliary microwave frequencies for pulse repetition rates, which transfers the noise contained in the microwave source into every comb tooth with a multiplication. For DKS microcombs, although self-referencing with broadband spectra has been achieved $(31,32)$, the typical line spacings of a few hundred gigahertz to a few terahertz are beyond the detection bandwidth of conventional electronics, which is undesirable in many applications. Recently, a dual-microcomb scheme was demonstrated (32), using a second microcomb with a small line spacing to bridge the wide gap $(\sim 1 \mathrm{THz})$ of an octave spanning microcomb (33). Despite its successful implementation in optical frequency synthesis, such a scheme requires sophisticated active locking systems, and the fixed line spacing of the adopted microcomb could limit the wider application when a varied line spacing is needed.

In this work, we present a novel frequency division scheme by injecting a soliton microcomb with a repetition frequency of $f_{\text {rep }}$ into a distributed feedback (DFB) laser that is operating in the gain switching mode. By optical injection locking the DFB laser with the microcomb and actively locking the gain switching frequency $\left(f_{\mathrm{gs}}\right)$ to a subharmonic frequency of the soliton repetition rate (i.e., $f_{\mathrm{gs}}=$ $f_{\text {rep }} / n$, with $n$ being a positive integer), the DFB laser emits a frequency comb that spans nearly $50 \mathrm{~nm}$, with a repetition frequency that is a perfect fraction of the soliton repetition rate. In contrast to the conventional optical frequency division technique that transfers the stability of an optical frequency into the microwave domain, our scheme transfers the spectral purity of the cavity soliton oscillator at $f_{\text {rep }}$ to the phase-locked $f_{\text {gs }}$ with a reduction of a factor of $n^{2}$ in the 
phase noise spectrum, even when $f_{\text {rep }}$ is too high to be directly measured with conventional electronics.

Figure 1A depicts the principle. A soliton microcomb generated by a CW laser-driven microresonator is injected into a GSL to provide the main comb lines and the phase coherence of the lasing field. In the meantime, the semiconductor laser is gain-switched at a frequency close to the subharmonic frequencies of the microcomb repetition rate $f_{\text {rep. }}$. The gain switching frequency is produced by a tunable signal generator such as a voltage-controlled oscillator (VCO). In the frequency domain, gain switching gives rise to sidebands around main comb lines (i.e., the emissions that are opticalinjection-locked to the master microcomb), and the beating between the sidebands and other main comb lines or their sidebands essentially causes laser intensity fluctuations that can be directly used as an error signal to implement subharmonic phase locking [SPL; see the Supplementary Materials for the physical model based on the laser rate equations $(25,34)$ and the Lugiato-Lefever equation (35)]. Figure 1C illustrates the SPL mechanism. The GSL spectral components denoted by the number 0 are the main comb lines. Gain switching at approximately $f_{\text {rep }} / n$ ( $n=2$ in the figure) generates multiple sidebands (whose orders are shown by the corresponding numbers). These sidebands produce multiple beat signals at frequencies of $\Delta f$ and its harmonics, which correspond to an intensity-modulated signal in the time domain (see the Supplementary Materials for the simulation of the error signals). The latter can be directly used for the control of the VCO frequency for the SPL. Once the SPL is activated, the beat frequencies of $\Delta f$ and its harmonics vanish, as the intensity error signal is locked to a constant level. Consequently, $f_{\mathrm{gs}}$ is controlled to be an exact fraction of $f_{\text {rep }}$, which is illustrated in Fig. 1E. Here, we note that the SPL cannot be implemented with the sideband generation method via electro-optic modulation (36) due to the lack of intensity modulation-induced error signals. Figure 1 (D and F) shows the experimentally measured GSL intensities in the time domain when the SPL is off and on, respectively. With the SPL being activated, the gain switching frequency is feedback-controlled to be exactly half of the soliton repetition rate of $14.09 \mathrm{GHz}$, and the emitted pulse train is highly coherent as depicted in Fig. 1F, exhibiting substantially reduced intensity jitter.

\section{RESULTS}

In the first experiment (see Fig. 2A for the setup), a crystalline magnesium fluoride $\left(\mathrm{MgF}_{2}\right)$ whispering gallery mode resonator with a cavity free spectral range (FSR) of $14.09 \mathrm{GHz}$ is used to generate the DKS. The pump-resonance detuning is stabilized with the PoundDrever-Hall (PDH) sideband locking technique (37). The soliton microcomb is amplified to an average power of $5 \mathrm{~mW}$ with an erbiumdoped fiber amplifier before it is injected into the GSL. The GSL outputs an average power of $\sim 1 \mathrm{~mW}$, half of which is sent to an optical spectrum analyzer (OSA) for spectrum acquisition. The other half of the light is registered by a photodetector with a bandwidth of $\sim 5 \mathrm{MHz}$, and the photodetector voltage is used as the error
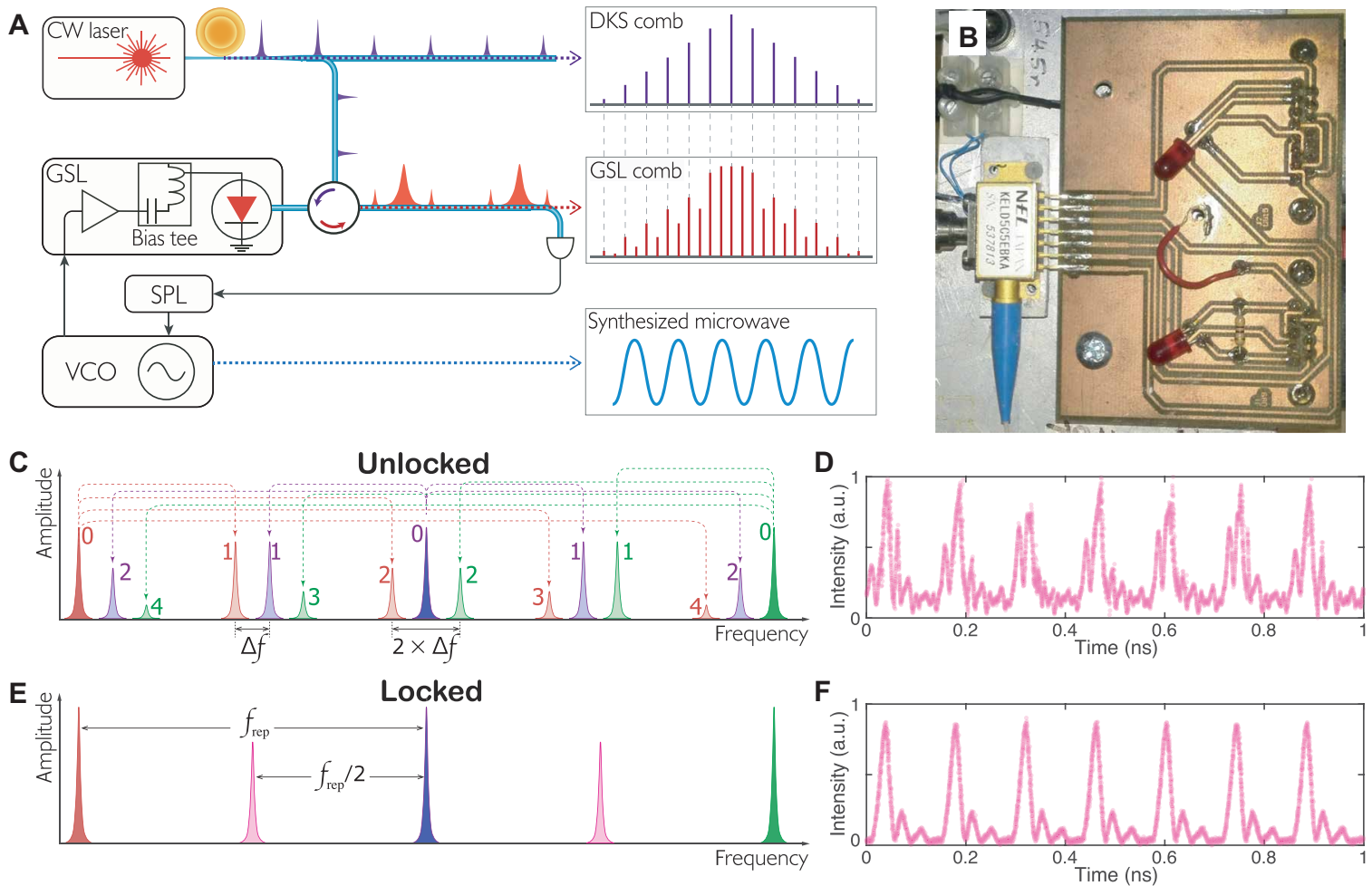

Fig. 1. Microcomb repetition rate division with a soliton-injected GSL. (A) The conceptual illustration of the scheme. The VCO is controlled by the SPL servo, outputting microwave frequency that is a subharmonic of the soliton repetition rate. (B) A photograph of the DFB laser used in this work. (C) Illustration of the gain-switched comb when the SPL is off. Multiple frequencies caused by the beating between sidebands of different microcomb teeth are generated. (D) The output of a soliton-injected GSL (with the SPL off) measured by an optical sampling oscilloscope. a.u., arbitrary units. (E) Illustration of the subharmonic phase-locked scenario in the frequency domain. (F) Output of the GSL in the time domain when it is subharmonic phase-locked to the soliton repetition rate (14.09 GHz). Photo credit: Wenle Weng, EPFL. 
A
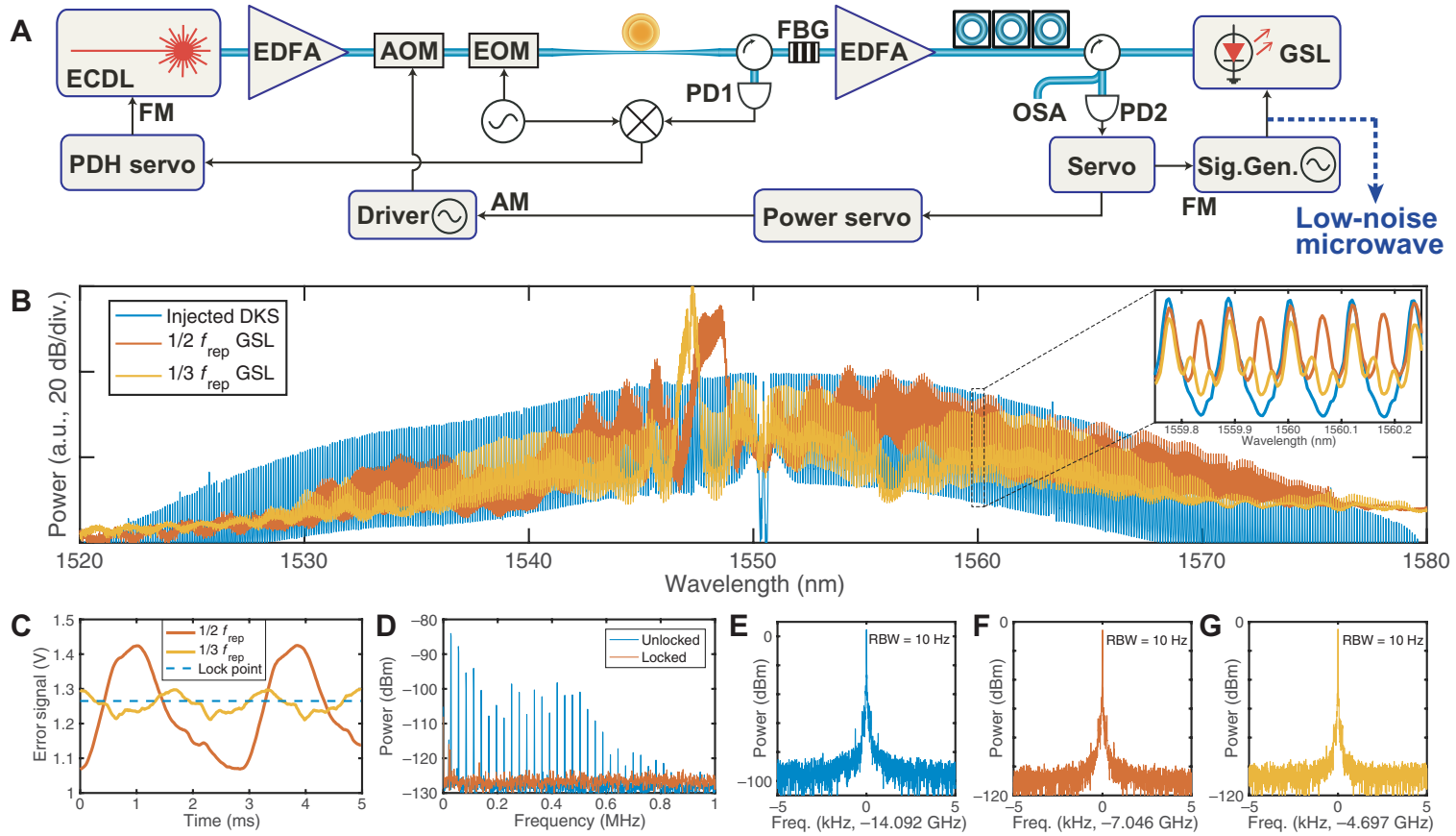

Fig. 2. Frequency division of a soliton microcomb generated by a crystalline microresonator. (A) Experimental setup. A fiber Bragg grating (FBG) and a circulator are used to separate the pumping external cavity diode laser (ECDL) from the solitons. A photodetector (PD1) and an electro-optic modulator (EOM) are used to produce PDH signals to lock the laser-resonance detuning. Half of the GSL power is registered by a photodetector (PD2) to produce subharmonic locking error signals. A servo locks the

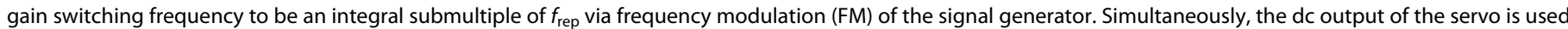
as an error signal to stabilize $f_{\text {rep }}$ through amplitude modulation (AM) of the pump laser power with an acousto-optic modulator (AOM). (B) The optical spectra of the microcomb and the soliton-injected GSL gain-switched at $f_{\text {rep }} / 2$ and $f_{\text {rep }} / 3$, respectively. The inset shows an enlargement of a portion of the spectra. (C) The error signals generated by PD2. The dashed line indicates the locking point. (D) The power spectra of the soliton-injected GSL when the SPL is on (red) and off (blue). (E to G) The spectra of DKS $f_{\text {rep }}$ and the phase-locked gain switching frequencies at $f_{\text {rep }} / 2$ and $f_{\text {rep }} / 3$, respectively.

signal to implement the SPL. In this proof-of-principle demonstration, we use a microwave signal generator to generate $f_{\mathrm{gs}}$. The error signal is fed back with a proportional-integral control servo to the frequency modulation port of the signal generator. To suppress the long-term drift of $f_{\text {rep }}$ that is mainly caused by the temperature drift of the microresonator, we use the dc level of the SPL servo correction signal as the error signal to control the pump laser intensity with a power servo whose control bandwidth is $\sim 10 \mathrm{~Hz}$. One should note that this power servo is not necessary for the SPL system and that $f_{\mathrm{gs}}$ can be effectively locked to the subharmonic frequencies of $f_{\text {rep }}$ even when $f_{\text {rep }}$ drifts for more than $1 \mathrm{MHz}$ (see the Supplementary Materials for the long-term frequency instabilities). Two gain switching frequencies at $f_{\text {rep }} / 2(7.05 \mathrm{GHz})$ and $f_{\text {rep }} / 3(4.70 \mathrm{GHz})$ are applied, respectively. Figure 2B presents the optical spectra of the amplified microcomb and the GSL OFC. The soliton-injected GSL spectra show prominent emissions at frequencies around $1548 \mathrm{~nm}$, with a span of a few nanometers. The comb spectra span over nearly $50 \mathrm{~nm}$ with less intensive teeth that exhibit perfect repetition rate division of the DKS microcomb, despite the fact that amplitude nonuniformities are presented (see the Supplementary Materials for the possible reasons). Figure $2 \mathrm{C}$ shows the error signals generated by the photodetector (PD2) for the SPL servo. With $f_{\mathrm{gs}}$ at $7.05 \mathrm{GHz}$, the error signal shows great contrast. For $f_{\mathrm{gs}}=4.70 \mathrm{GHz}$, the contrast of the error signal deteriorates as the GSL pulse width increases with decreased gain switching frequency, leading to reduced sideband intensities. Such inefficiency of gain switching can also be ob- served from the significantly lower amplitude of the gain switching sidebands in the comb spectrum (see the inset of Fig. 2B). Still, owing to the excellent relative intensity noise (RIN) performance of the GSL (38), the SPL is robust and can be sustained indefinitely.

We measure the spectral qualities of the synthesized $f_{\mathrm{gs}}$ signals and $f_{\text {rep }}$ that is generated by the photodetection of the DKS train with a fast photodetector (bandwidth of $\sim 30 \mathrm{GHz}$ ). Figure 2 (E to G) shows these signals obtained with an electrical spectrum analyzer. In Fig. 3, we present the phase noise spectra of these signals. Within the SPL servo control bandwidth of $\sim 10 \mathrm{kHz}$, the phase noise levels of the synthesized $f_{\mathrm{gs}}$ are exceptionally lower than the intrinsic phase noise levels of the signal generator. Moreover, they are lower than that of $f_{\text {rep }}$ by 6 and $9.5 \mathrm{~dB}$, respectively. Such a reduction corresponds to a factor of $n^{2}$ improvement with $n=2$ for $7.05 \mathrm{GHz}$ and $n=3$ for $4.70 \mathrm{GHz}$, showing the expected benefit of frequency division. Reaching a level below $-115 \mathrm{dBc} / \mathrm{Hz}$ at the offset frequency of $1 \mathrm{kHz}$, the low-phase noise of the synthesized microwaves is already comparable with, or better than, the state-of-the-art performance of parity time symmetric optoelectronic oscillators $(39,40)$, microphotonic microwave synthesizers based on stimulated Brillouin oscillations (41), and electro-optical frequency division (42).

Next, we apply our frequency division scheme to detect and divide a high soliton repetition rate that cannot be conveniently measured with our electronic instruments. We use a silicon nitride $\left(\mathrm{Si}_{3} \mathrm{~N}_{4}\right)$ microring resonator with an FSR of $\sim 100 \mathrm{GHz}$ for DKS generation. The gain switching is implemented at three different frequencies of 
$16.82,10.09$, and $6.73 \mathrm{GHz}$, corresponding to a division factor of $n=6,10$, and 15 , respectively. The amplified DKS microcomb spectrum and the GSL spectra are displayed in Fig. 4 (A and B). The GSL OFC shows equally spaced teeth filling the gaps between the microcomb teeth. The GSL comb teeth are well resolved with a resolution limited by that of the OSA, showing that the GSL maintains excellent coherence (see the Supplementary Materials for corroborating optical heterodyne measurement). In Fig. 4C, we plot the phase noise spectra of the synthesized $f_{\mathrm{gs}}$ signals, based on which we infer

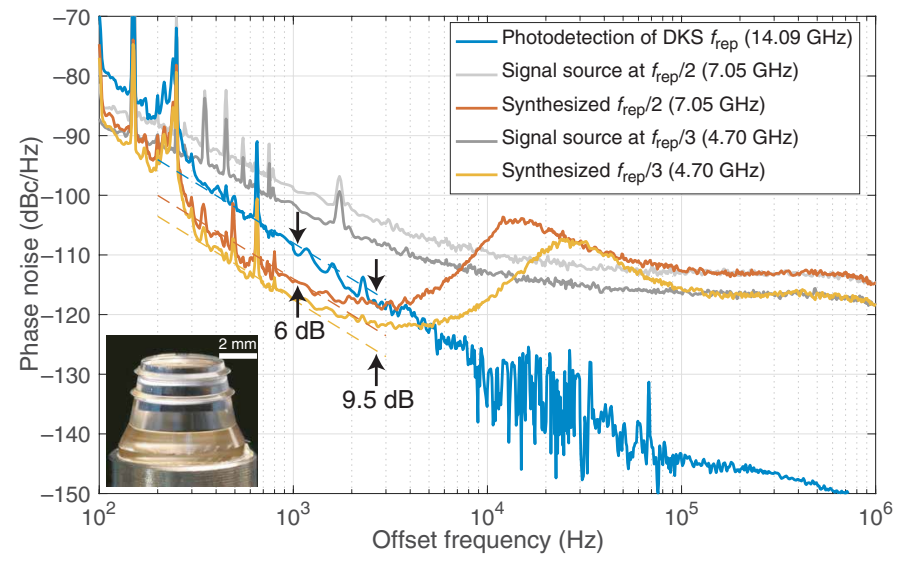

Fig. 3. Phase noise spectra of synthesized microwave signals. The $14.09-\mathrm{GHz}$ DKS $f_{\text {rep }}$ is generated by photodetection of the soliton train. The synthesized signals at $7.05\left(f_{\text {rep }} / 2\right)$ and $4.70 \mathrm{GHz}\left(f_{\text {rep }} / 3\right)$ are output by the frequency-modulated signal generator when the SPL is activated. The servo has a control bandwidth of $\sim 10 \mathrm{kHz}$, causing the rising noise level around that frequency. Within the control bandwidth, the noise levels of the two synthesized microwaves are lower than that of $f_{\text {rep }}$ by 6 and $9.5 \mathrm{~dB}$ (the dashed lines with a slope of $f^{-2}$ are plotted for visual guidance). At frequencies above $100 \mathrm{kHz}$, the phase noise of the synthesized microwaves are identical to the levels intrinsic to the free running signal generator because of the servo's limited bandwidth. The inset shows a photograph of the $\mathrm{MgF}_{2}$ resonator. Photo credit: Tobias Herr, EPFL. the phase noise of the 100.93-GHz $f_{\text {rep }}$. Here, we note that the phase noise performance of the synthesized microwaves is similar to that of the microwaves derived from the recently developed $\mathrm{X}$ - and $\mathrm{K}$-band $\mathrm{Si}_{3} \mathrm{~N}_{4}$ soliton microcomb oscillators (43). This phase noise level, which is much higher than the fundamental thermal noise limit (44), is attributed to technical noise such as ambient temperature fluctuations, pump laser phase noise, and RIN (43). With progress on the microresonator fabrication process and technical noise suppression in the future, we expect the phase noise to be considerably improved to enable on-chip low-noise millimeter-wave and microwave synthesizers based on GSL frequency division.

\section{DISCUSSION}

In summary, built upon the marriage between soliton microcomb and gain-switched comb, we present a hybrid photonic system that not only yields a broadband frequency comb with a line spacing equal to the divided soliton rate but also synthesizes low-noise signals in the microwave domain. In contrast to traditional optical injection locking method that uses a CW laser as the master, our scheme injection locks a semiconductor laser to the entire microcomb, transferring both the carrier phase coherence and the soliton repetition rate spectral purity to the GSL. As a result, with active control, the GSL can generate additional comb teeth that are fully coherent and equally spaced, thus facilitating the application of highrepetition rate microcombs in metrology and spectroscopy. In addition to low-noise microwave synthesis, this technique can also be used to measure the phase noise of pulse repetition rate that is beyond the detection bandwidth of conventional electronic devices. Moreover, with the optical pulse injection locking scheme, we are able to carry out OFC repetition frequency multiplication with the GSL (see the Supplementary Materials for more information). The versatility of the configuration highlights the joint effort by different OFC technologies. As both comb devices are chip-based, future endeavor can be made to the development of a fully integrated
A

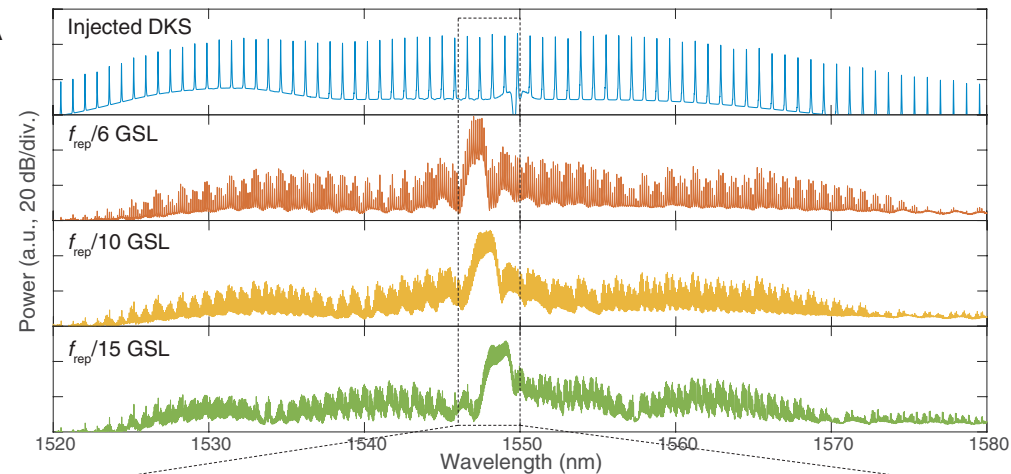

B

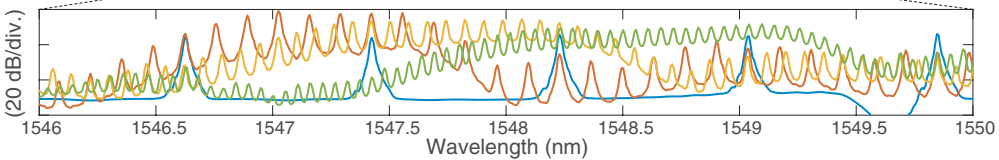

C

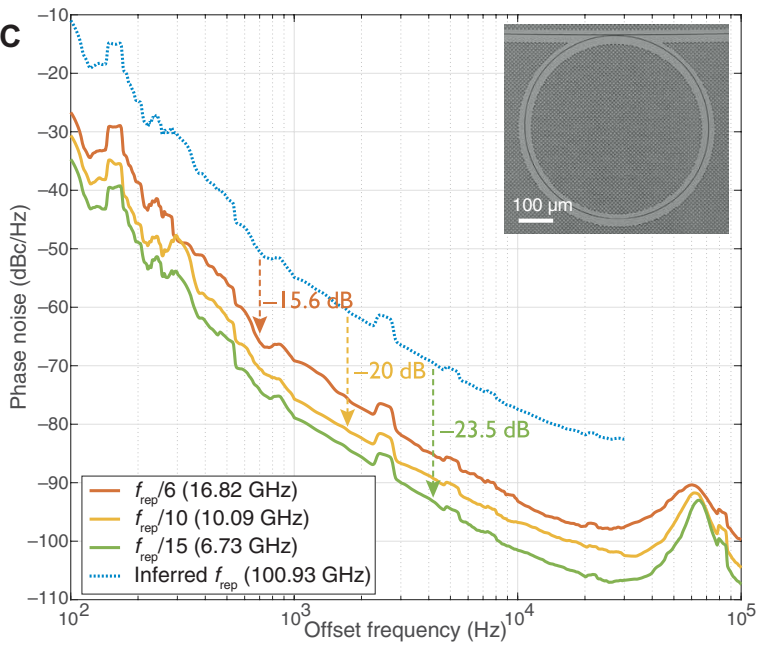

Fig. 4. Frequency division of a $\mathbf{1 0 0}-\mathbf{G H z}$ repetition rate photonic chip $\mathbf{S i}_{3} \mathbf{N}_{\mathbf{4}}$ microcomb. (A) From top to bottom: the optical spectra of the amplified soliton microcomb and the GSL with gain switching rates of $f_{\text {rep }} / 6, f_{\text {rep }} / 10$, and $f_{\text {rep }} / 15$, respectively. (B) The enlargement of the comb spectra from 1546 to $1550 \mathrm{~nm}$. (C) The phase noise spectra of the synthesized microwave signals. The blue dotted trace is the phase noise level of the 100-GHz $f_{\text {rep }}$ inferred from the measured phase noise levels of the synthesized signals. The trace is not plotted above $30 \mathrm{kHz}$ because of the limited control bandwidth of the SPL servo. The inset is a photograph of the $\mathrm{Si}_{3} \mathrm{~N}_{4}$ microresonator. 
miniaturized architecture that could find extensive applications in demanding environments.

\section{MATERIALS AND METHODS $\mathbf{M g F}_{\mathbf{2}}$ resonator}

The $\mathrm{MgF}_{2}$ resonator was fabricated by diamond turning a crystalline cylindrical blank and subsequent surface polishing. The intrinsic linewidth of the resonance is $\sim 100 \mathrm{kHz}$, corresponding to an intrinsic quality factor $(Q)$ of $\sim 2 \times 10^{9}$. The second-order dispersion coefficient $\left(\frac{D_{2}}{2 \pi}\right)$ at $1550 \mathrm{~nm}$ is approximately $2 \mathrm{kHz}$. To generate solitons, a tapered fiber is used to evanescently couple light into and out of the resonator. The pump laser is amplified to $\sim 100 \mathrm{~mW}$. Using the PDH sideband locking technique, the pump-resonance detuning is locked at $6 \mathrm{MHz}$.

\section{$\mathrm{Si}_{3} \mathrm{~N}_{4}$ resonator}

The $\mathrm{Si}_{3} \mathrm{~N}_{4}$ microring resonator was fabricated with the photonic Damascene process (45). The coupling section is designed to operate close to the critical coupling regime for $1550 \mathrm{~nm}$. The loaded linewidth $\left(\frac{\kappa}{2 \pi}\right)$ of the resonance is $\sim 30 \mathrm{MHz}$, corresponding to a loaded $Q$ of $\sim 6.7 \times 10^{6}$. The dispersion parameter $\left(\frac{D_{2}}{2 \pi}\right)$ is measured to be $\sim 1 \mathrm{MHz}$. Fiber lenses were used to couple light into and out of the waveguide that is evanescently coupled to the microring. The pump laser power is $80 \mathrm{~mW}$. The pump-resonance detuning is locked at $300 \mathrm{MHz}$ with the $\mathrm{PDH}$ sideband locking technique.

\section{Semiconductor GSL}

The laser used for generation of the GSL OFC is a commercially available DFB laser (NTT Electronics) encased in a seven-pin butterfly package, without an output isolator. The laser had a threshold current of $10 \mathrm{~mA}$ and $18-\mathrm{GHz}$ modulation bandwidth. The gain switching is achieved by combining, via a bias tee, the dc bias current of $\sim 50 \mathrm{~mA}$ with an RF signal (after amplification with an RF amplifier) from a microwave signal generator (Rohde\&Schwarz SMB100A). A fiber circulator is used to inject the soliton microcomb into the laser. The polarization state of the injected light was adjusted by a fiber polarization controller to optimize the contrast of the error signal for the SPL.

\section{SUPPLEMENTARY MATERIALS}

Supplementary material for this article is available at http://advances.sciencemag.org/cgi/ content/full/6/39/eaba2807/DC1

\section{REFERENCES AND NOTES}

1. T. Udem, R. Holzwarth, T. W. Hänsch, Optical frequency metrology. Nature 416, 233-237 (2002).

2. S. T. Cundiff, J. Ye, Colloquium: Femtosecond optical frequency combs. Rev. Mod. Phys. 75, 325 (2003).

3. G. Porat, C. M. Heyl, S. B. Schoun, C. Benko, N. Dörre, K. L. Corwin, J. Ye, Phasematched extreme-ultraviolet frequency-comb generation. Nat. Photonics 12, 387-391 (2018).

4. A. V. Muraviev, V. O. Smolski, Z. E. Loparo, K. L. Vodopyanov, Massively parallel sensing of trace molecules and their isotopologues with broadband subharmonic mid-infrared frequency combs. Nat. Photonics 12, 209-214 (2018).

5. I. Ushijima, M. Takamoto, M. Das, T. Ohkubo, H. Katori, Cryogenic optical lattice clocks. Nat. Photonics 9, 185-189 (2015).

6. S. L. Campbell, R. B. Hutson, G. E. Marti, A. Goban, N. Darkwah Oppong, R. L. McNally, L. Sonderhouse, J. M. Robinson, W. Zhang, B. J. Bloom, J. Ye, A Fermi-degenerate three-dimensional optical lattice clock. Science 358, 90-94 (2017).
7. S. B. Koller, J. Grotti, S. Vogt, A. Al-Masoudi, S. Dörscher, S. Häfner, U. Sterr, C. Lisdat, Transportable optical lattice clock with $7 \times 10^{-17}$ uncertainty. Phys. Rev. Lett. 118, 073601 (2017).

8. J. G. Hartnett, C. R. Locke, E. N. Ivanov, M. E. Tobar, P. L. Stanwix, Cryogenic sapphire oscillator with exceptionally high long-term frequency stability. Appl. Phys. Lett. 89, 203513 (2006).

9. E. N. Ivanov, M. E. Tobar, Low phase-noise sapphire crystal microwave oscillators: Current status. IEEE Trans. Ultrason. Ferroelectr. Freq. Control 56, 263-269 (2009).

10. N. Picqué, T. W. Hänsch, Frequency comb spectroscopy. Nat. Photonics 13, 146-157 (2019).

11. T. M. Fortier, M. S. Kirchner, F. Quinlan, J. Taylor, J. C. Bergquist, T. Rosenband, N. Lemke, A. Ludlow, Y. Jiang, C. W. Oates, S. A. Diddams, Generation of ultrastable microwaves via optical frequency division. Nat. Photonics 5, 425-429 (2011).

12. F. N. Baynes, F. Quinlan, T. M. Fortier, Q. Zhou, A. Beling, J. C. Campbell, S. A. Diddams, Attosecond timing in optical-to-electrical conversion. Optica 2, 141-146 (2015).

13. A. L. Gaeta, M. Lipson, T. J. Kippenberg, Photonic-chip-based frequency combs. Nat. Photonics 13, 158-169 (2019).

14. T. J. Kippenberg, R. Holzwarth, S. A. Diddams, Microresonator-based optical frequency combs. Science 332, 555-559 (2011).

15. M. Imran, P. M. Anandarajah, A. Kaszubowska-Anandarajah, N. Sambo, L. Potí, A survey of optical carrier generation techniques for terabit capacity elastic optical networks. IEEE Commun. Surveys Tutor. 20, 211-263 (2017).

16. T. J. Kippenberg, A. L. Gaeta, M. Lipson, M. L. Gorodetsky, Dissipative Kerr solitons in optical microresonators. Science 361, eaan8083 (2018).

17. P. Trocha, M. Karpov, D. Ganin, M. H. P. Pfeiffer, A. Kordts, S. Wolf, J. Krockenberger, P. Marin-Palomo, C. Weimann, S. Randel, W. Freude, T. J. Kippenberg, C. Koos, Ultrafast optical ranging using microresonator soliton frequency combs. Science 359, 887-891 (2018).

18. M.-G. Suh, K. J. Vahala, Soliton microcomb range measurement. Science 359, 884-887 (2018).

19. W. Liang, D. Eliyahu, V. S. Ilchenko, A. A. Savchenkov, A. B. Matsko, D. Seidel, L. Maleki, High spectral purity Kerr frequency comb radio frequency photonic oscillator. Nat. Commun. 6, 7957 (2015).

20. Z. L. Newman, V. Maurice, T. Drake, J. R. Stone, T. C. Briles, D. T. Spencer, C. Fredrick, Q. Li, D. Westly, B. R. Ilic, B. Shen, M.-G. Suh, K. Y. Yang, C. Johnson, D. M. S. Johnson, L. Hollberg, K. J. Vahala, K. Srinivasan, S. A. Diddams, J. Kitching, S. B. Papp, M. T. Hummon, Architecture for the photonic integration of an optical atomic clock. Optica 6, 680-685 (2019).

21. J. van der Ziel, R. Logan, Generation of short optical pulses in semiconductor lasers by combined DC and microwave current injection. IEEE J. Quantum Electron. 18, 1340-1350 (1982).

22. P. Paulus, R. Langenhorst, D. Jager, Generation and optimum control of picosecond optical pulses from gain-switched semiconductor lasers. IEEE J. Quantum Electron. 24 1519-1523 (1988)

23. P. M. Anandarajah, C. Guignard, A. Clarke, D. Reid, M. Rensing, L. P. Barry, G. Edvell, J. D. Harvey, Optimized pulse source employing an externally injected gain-switched laser diode in conjunction with a nonlinearly chirped grating. IEEE J. Sel. Top. Quantum Electron. 12, 255-264 (2006).

24. R. Zhou, S. Latkowski, J. O'Carroll, R. Phelan, L. P. Barry, P. Anandarajah, $40 \mathrm{~nm}$ wavelength tunable gain-switched optical comb source. Opt. Express 19, B415-B420 (2011).

25. S. P. Ó Dúill, P. M. Anandarajah, R. Zhou, L. P. Barry, Numerical investigation into the injection-locking phenomena of gain switched lasers for optical frequency comb generation. Appl. Phys. Lett. 106, 211105 (2015).

26. A. Rosado, A. Pérez-Serrano, J. M. G. Tijero, Á. Valle, L. Pesquera, I. Esquivias, Enhanced optical frequency comb generation by pulsed gain-switching of optically injected semiconductor lasers. Opt. Express 27, 9155-9163 (2019).

27. L. C. Comandar, M. Lucamarini, B. Fröhlich, J. F. Dynes, A. W. Sharpe, S. W.-B. Tam, Z. L. Yuan, R. V. Penty, A. J. Shields, Quantum key distribution without detector vulnerabilities using optically seeded lasers. Nat. Photonics 10, 312-315 (2016).

28. Z. L. Yuan, M. Lucamarini, J. F. Dynes, B. Fröhlich, A. Plews, A. J. Shields, Robust random number generation using steady-state emission of gain-switched laser diodes. Appl. Phys. Lett. 104, 261112 (2014).

29. H. Shams, T. Shao, M. J. Fice, P. M. Anandarajah, C. C. Renaud, F. Van Dijk, L. P. Barry, A. J. Seeds, $100 \mathrm{~Gb} / \mathrm{s}$ multicarrier $\mathrm{THz}$ wireless transmission system with high frequency stability based on a gain-switched laser comb source. IEEE Photonics J. 7, 1-11 (2015).

30. M. D. G. Pascual, V. Vujicic, J. Braddell, F. Smyth, P. Anandarajah, L. Barry, Photonic integrated gain switched optical frequency comb for spectrally efficient optical transmission systems. IEEE Photonics J. 9, 1-8 (2017).

31. V. Brasch, E. Lucas, J. D. Jost, M. Geiselmann, T. J. Kippenberg, Self-referenced photonic chip soliton Kerr frequency comb. Light Sci. Appl. 6, e16202 (2017).

32. D. T. Spencer, T. Drake, T. C. Briles, J. Stone, L. C. Sinclair, C. Fredrick, Q. Li, D. Westly, B. R. Ilic, A. Bluestone, N. Volet, T. Komljenovic, L. Chang, S. H. Lee, D. Y. Oh, M.-G. Suh, K. Y. Yang, M. H. P. Pfeiffer, T. J. Kippenberg, E. Norberg, L. Theogarajan, K. Vahala, N. R. Newbury, K. Srinivasan, J. E. Bowers, S. A. Diddams, S. B. Papp, An optical-frequency synthesizer using integrated photonics. Nature 557, 81-85 (2018). 
33. T. C. Briles, J. R. Stone, T. E. Drake, D. T. Spencer, C. Fredrick, Q. Li, D. Westly, B. R. llic, K. Srinivasan, S. A. Diddams, S. B. Papp, Interlocking Kerr-microresonator frequency combs for microwave to optical synthesis. Opt. Lett. 43, 2933-2936 (2018).

34. N. Schunk, K. Petermann, Noise analysis of injection-locked semiconductor injection lasers. IEEE J. Quantum Electron. 22, 642-650 (1986).

35. L. A. Lugiato, R. Lefever, Spatial dissipative structures in passive optical systems. Phys. Rev. Lett. 58, 2209 (1987).

36. P. Del'Haye, S. B. Papp, S. A. Diddams, Hybrid electro-optically modulated microcombs. Phys. Rev. Lett. 109, 263901 (2012).

37. W. Weng, E. Lucas, G. Lihachev, V. E. Lobanov, H. Guo, M. L. Gorodetsky, T. J. Kippenberg, Spectral purification of microwave signals with disciplined dissipative Kerr solitons. Phys. Rev. Lett. 122, 013902 (2019).

38. M. D. G. Pascual, R. Zhou, F. Smyth, P. M. Anandarajah, L. P. Barry, Software reconfigurable highly flexible gain switched optical frequency comb source. Opt. Express 23, 23225-23235 (2015).

39. J. Zhang, J. Yao, Parity-time-symmetric optoelectronic oscillator. Sci. Adv. 4, eaar6782 (2018).

40. H. Tang, Y. Yu, X. Zhang, Widely tunable optoelectronic oscillator based on selective parity-time-symmetry breaking. Optica 6, 944-950 (2019).

41. J. Li, H. Lee, K. J. Vahala, Microwave synthesizer using an on-chip Brillouin oscillator. Nat. Commun. 4, 2097 (2013).

42. J. Li, X. Yi, H. Lee, S. A. Diddams, K. J. Vahala, Electro-optical frequency division and stable microwave synthesis. Science 345, 309-313 (2014)

43. J. Liu, E. Lucas, A. S. Raja, J. He, J. Riemensberger, R. N. Wang, M. Karpov, H. Guo, R. Bouchand, T. J. Kippenberg, Photonic microwave generation in the X-and K-band using integrated soliton microcombs. Nat. Photonics 14, 486-491 (2020).

44. G. Huang, E. Lucas, J. Liu, A. S. Raja, G. Lihachev, M. L. Gorodetsky, N. J. Engelsen, T. J. Kippenberg, Thermorefractive noise in silicon-nitride microresonators. Phys. Rev. A 99, 061801(R) (2019).
45. M. H. P. Pfeiffer, A. Kordts, V. Brasch, M. Zervas, M. Geiselmann, J. D. Jost, T. J. Kippenberg, Photonic Damascene process for integrated high-Q microresonator based nonlinear photonics. Optica 3, 20-25 (2016).

Acknowledgments: We thank R. Bouchand and E. Lucas for useful discussions and technical assistance. Funding: This publication was supported by Contract D18AC00032 (DRINQS) from the Defense Advanced Research Projects Agency (DARPA), Defense Sciences Office (DSO), funding from the Swiss National Science Foundation under grant agreement No. 192293, Science Foundation Ireland (SFI) 15/CDA/3640, and the SFI/European Regional Development Fund (13/RC/2077). Author contributions: W.W. and A.K.-A. conceived the project. W.W. carried out the experiment, analyzed the data, developed the theoretical model, and performed the simulation. A.K.-A. and P.M.A. developed the GSL. J.L. fabricated the $\mathrm{Si}_{3} \mathrm{~N}_{4}$ resonator. W.W. wrote the manuscript, with input from all the other authors. T.J.K. and P.M.A. supervised the project. Competing interests: The authors declare that they have no competing interests. Data and materials availability: All data needed to evaluate the conclusions in the paper are present in the paper and/or the Supplementary Materials. The data and code used to produce the results of this manuscript are available on Zenodo: [https:// doi.org/10.5281/zenodo.3876291]. Additional data related to this paper may be requested from the authors.

Submitted 23 November 2019

Accepted 14 August 2020

Published 25 September 2020

10.1126/sciadv.aba2807

Citation: W. Weng, A. Kaszubowska-Anandarajah, J. Liu, P. M. Anandarajah, T. J. Kippenberg, Frequency division using a soliton-injected semiconductor gain-switched frequency comb. Sci. Adv. 6, eaba2807 (2020). 


\section{ScienceAdvances}

Frequency division using a soliton-injected semiconductor gain-switched frequency comb Wenle Weng, Aleksandra Kaszubowska-Anandarajah, Junqiu Liu, Prince M. Anandarajah and Tobias J. Kippenberg

Sci Adv 6 (39), eaba2807.

DOI: $10.1126 /$ sciadv.aba2807

ARTICLE TOOLS

SUPPLEMENTARY

MATERIALS

REFERENCES

PERMISSIONS http://advances.sciencemag.org/content/6/39/eaba2807

http://advances.sciencemag.org/content/suppl/2020/09/21/6.39.eaba2807.DC1

This article cites 45 articles, 7 of which you can access for free http://advances.sciencemag.org/content/6/39/eaba2807\#BIBL

http://www.sciencemag.org/help/reprints-and-permissions

Science Advances (ISSN 2375-2548) is published by the American Association for the Advancement of Science, 1200 New York Avenue NW, Washington, DC 20005. The title Science Advances is a registered trademark of AAAS.

Copyright @ 2020 The Authors, some rights reserved; exclusive licensee American Association for the Advancement of Science. No claim to original U.S. Government Works. Distributed under a Creative Commons Attribution NonCommercial License 4.0 (CC BY-NC). 